\title{
Potential role of leptin in angiogenesis: leptin induces endothelial cell proliferation and expression of matrix metalloproteinases in vivo and in vitro
}

\author{
Hyun-Young Park ${ }^{1 *}$, Hyuck Moon Kwon ${ }^{1,2,3,4 *}$, \\ Hyun Joung Lim ${ }^{1,2,3}$, Bum Kee Hong ${ }^{1,2}$, \\ Ju Yong Lee ${ }^{2}$, Byoung Eun Park ${ }^{2}$, \\ Yangsoo Jang ${ }^{1,2,3}$, Seung Yun $\mathrm{Cho}^{2,3}$ \\ and Hyun-Seung Kim ${ }^{2}$ \\ ${ }^{1}$ Yonsei Cardiovascular Research Institute, Yonsei University \\ College of Medicine, Seoul, Korea \\ ${ }^{2}$ Cardiology Division, Department of Internal Medicine, \\ Yonsei University College of Medicine, Seoul, Korea \\ ${ }^{3}$ BK21 Project for Medical Science, Yonsei University \\ College of Medicine, Seoul, Korea \\ ${ }^{4}$ Corresponding author: Tel, +82-2-3497-3316, 3330; \\ Fax, +82-2-573-0112; E-mail, kwonhm@yumc.yonsei.ac.kr \\ *These authors contributed equally to this work.
}

Accepted 26 June 2001

Abbreviations: ox-LDL, oxidized low density lipoprotein; SD rat, Sprague-Dawley rat; SEM, scanning electron microscopy; PBS, phosphate buffered saline

\begin{abstract}
Leptin, the product of ob gene, is an endocrine hormone that regulates adipose tissue mass. Recently, leptin has been found to generate a growth signal involving a tyrosine kinase-dependent intracellular pathway and promote angiogenic processes via activation of leptin receptor (Ob-R) in endothelial cells. However, it is not clear how leptin functions to promote multi-step processes involved in the neovascularization at the atherosclerotic plaque. We have examined the expression of matrix metalloproteinases (MMPs) and tissue inhibitors of metalloproteinases (TIMPs) and Ob-R in human atherosclerotic lesions, leptin-mediated angiogenesis in vivo and in vitro. Immunohistochemical analysis of human atherosclerotic aorta revealed an increased expression of Ob-R in the intima of neorevascularized regions and of both MMPs and TIMPs predominantly in the endothelial lining of intimal neovessels and macrophages/foam cells. In the rat corneal angiogenesis assay, leptin elicited a comparable sensitivity of angiogenic activity to those of vascular endothelial growth factor (VEGF). The immunohistological anal-
\end{abstract}

ysis of the leptin-treated rat cornea showed definitive rises in Ob-R, MMPs and TIMPs expression as well as those of VEGF receptor (VEGFR-1). Leptin $(10-40 \mathrm{ng} / \mathrm{ml})$ induced proliferation of the human umbilical vein endothelial cells (HUVECs) and elevation of MMP-2, MMP-9, TIMP-1, and TIMP-2 expression in a dose-dependent manner. Leptin also induced increases of MMP-2, MMP-9, TIMP-1, and Up-regulated the human coronary artery smooth muscle cells (HCASMCs). These findings suggest that leptin, a hormone with pluralistic properties including a mitogenic activity on vascular endothelial cells, plays a role in matrix remodeling by regulating the expression of MMPs and TIMPs. Taken together, our findings further provide evidences for leptin's role as an angiogenesis inducer in the normal organ (rat cornea) and in aberrant vasculature under duress like atherosclerosis.

Keywords: leptin, angiogenesis, MMPs, atherosclerosis

\section{Introduction}

Leptin, the product of ob gene secreted by adipocytes (Campfield et al., 1995; Halaas et al., 1995; Pelleymounter et al., 1995), has been known to generate a growth signal involving a tyrosine kinase-dependent intracellular pathway and act as a functional link between adipocytes and the vasculature. Recently, treatment of HUVECs with leptin enhanced formation of capillary-like tubes in an in vitro angiogenesis assay and neovascularization in an in vivo model of angiogenesis (Bouloumié et al., 1998). Atherosclerotic diseases are known to be closely associated with high levels of LDL and obese state of patient and animals. However, any involvement of leptin in the progression of atherosclerotic diseases has not been well understood although neovascularization within the plaque has been known to be essential in the atheromatous plaque formation (Kahlon et al., 1992; O'Brien et al., 1994).

It is not clear whether a circulating leptin could exert paracrine effects on the plaque forming cells and participate in the progression of atherosclerosis. We have investigated the effects of leptin on vascularization in vitro and in vivo and on the microenvironment around the plaque by examining expression of tissue modeling enzymes (MMPs) and inhibitors (TIMPs). We have compared the effects of leptin in the aberrant 
tissue by examining the expression of Ob-R, VEGF and MMPs/TIMPs in atheromatous plaques and in normal tissue using angiogenesis-sensitive rat corneal model.

\section{Materials and Methods}

\section{Cell culture and proliferation assay}

Normal HUVECs and HCASMCs were purchased from Clonetics and maintained in SmGM2 and EGMMV bullet kits (Clonetics). HUVECs and HCASMCs from passage 5 to 13 were used in this study. For the proliferation assay, the cells in 24 wells were arrested for $24 \mathrm{~h}$ in serum-free medium and then incubated in serum-deprived medium supplemented with $2 \%$ FBS for $72 \mathrm{~h}$ in the presence of increasing concentration of leptin (10 to $40 \mathrm{ng} / \mathrm{ml}$ ). Total cell number was counted after trypsinization using a cell counter.

\section{In vitro angiogenesis assay}

Before the HUVECs were plated, the 24 well plates coated with growth factor-reduced basement membrane proteins (Matrigel, $0.3 \mathrm{ml} /$ well) were solidified at $37^{\circ} \mathrm{C}$ for $1 \mathrm{~h}$. Leptin was added to Matrigel solution prior to polymerization. For angiogenesis assay with HUVECs, the trypsinized cells were plated on the surface of Matrigel and cultured in serum-deprived medium $\left(8 \times 10^{4}\right.$ cells/well).

\section{Measurement of VEGF $_{165}$ and bFGF by ELISA}

Expression of VEGF 165 and bFGF by HUVECS and HCASMCs after stimulation of leptin (10 to $40 \mathrm{ng} / \mathrm{ml}$ ) were measured by ELISA kit (R\&D). The $100 \mathrm{ul}$ of standard or cultured medium was added into antibodycoated wells of the microplate and incubated at $25^{\circ} \mathrm{C}$ for $2 \mathrm{~h}$. The wells were washed 4 times with washing buffer and incubated at $25^{\circ} \mathrm{C}$ for $2 \mathrm{~h}$. The substrates were added to the washed well and reacted for $20 \mathrm{~min}$ at $25^{\circ} \mathrm{C}$. After adding the $50 \mathrm{ul}$ of stop buffer, the concentrations of $\mathrm{VEGF}_{165}$ and bFGF were measured with spectrophotometric reader at $450 \mathrm{~nm}$.

\section{Zymography}

Enzymatic activities of MMP-2 and MMP-9 were investigated using zymography analysis. The protein content of the culture medium was measured by the method of Bradford using bovine serum albumin as standard. Aliquots of culture media containing $50 \mathrm{ug}$ of protein from HUVECs and HCASMCs, with or without leptin treatment, were loaded on an 11\% SDS-PAGE gel containing $0.1 \%$ gelatin for electrophoresis. Gels were reacted with collagenase buffer for $16 \mathrm{~h}$ at $37^{\circ} \mathrm{C}$, stained with $0.25 \%$ Coomassie brilliant blue, and destained with $30 \%$ isopropanol in $10 \%$ acetic acid to visualize the MMP bands.

\section{Western blot analysis}

Fifty ug of protein was subjected to $8-12 \%$ gradient SDS-PAGE gel and transferred to immobilon-P membrane (Millipore, Bedford, MA) at $12 \mathrm{~V}$ for $1 \mathrm{~h}$. The membrane was blocked in $5 \%$ non-fat dry milk in TBS$\mathrm{T}$ at $25^{\circ} \mathrm{C}$ for $1 \mathrm{~h}$. TIMP-2 antibody $(5 \mathrm{ug} / \mathrm{ml}, 1: 2000$ dilution) and secondary antibody (goat anti-rabbit/mouse IgG, horseradish peroxidase-conjugated, Amersham) was used for the detection of TIMP-2. Signals were detected with an ECL kit (Amersham), and exposed to X-ray film (Kodak).

\section{Analysis of the expression of MMPs by reverse transcriptase-polymerase chain reaction (RT-PCR)}

Total RNA was extracted from cultured cells and $5 \mu \mathrm{g}$ of purified total RNA was incubated with reverse transcriptase, dNTP, random hexamer and reaction buffer in a final volume of $20 \mathrm{ul}$ at $37^{\circ} \mathrm{C}$ for $1 \mathrm{~h}$. After a final denaturation at $94^{\circ} \mathrm{C}$ for $10 \mathrm{~min}, 10 \mathrm{ul}$ of cDNA was subjected to PCR consisting of a denaturation at $95^{\circ} \mathrm{C}$ for $1 \mathrm{~min}$, followed by $1 \mathrm{~min}$ of annealing at $55^{\circ} \mathrm{C}$ and 1 min of elongation at $72^{\circ} \mathrm{C}$ for 35 cycles. The primers for amplification of MMP-1, $-2,-9$ and TIMP-1 were as follows; MMP-1: sense 5'-ggaaaacacatggtgtgagtcc-3', antisense: 5'-gttagaagagttatcccttgcc-3'; MMP-2: sense 5'-caggctcttctcctttcacaac-3', antisense 5'-aagccacggcttggtttcctc-3'; MMP-9: sense 5'-tgggctacgtgacctatgacat-3', antisense 5'-gcccagcccacctccactcctc-3'; TIMP-1: sense 5'-acccacagacggccttctgcaattc-3', antisense 5'-ggctatctgggaccgcagggactgc-3'. The amplified cDNA was size fractionated by agarose gel electrophoresis.

\section{In vivo angiogenesis assay}

Adult SD rats were anesthesized with ketamine and the eye was topically anesthesized with oxybuprocaine. With the use of an operating microscope, a central intrastromal linear keratotomy was performed with a surgical blade. A lamellar micropocket was then dissected and extended to within $2 \mathrm{~mm}$ of the superior limbus. Dehydrated pellet ( $2 \mathrm{~mm}$ diameter) made of $70 \%$ hydratable hydrogel containing PBS $(n=4)$ or $125 \mathrm{ng}$ of leptin $(n=4)$ were placed into the preformed corneal pocket. The eyes were routinely examined by slit lamp biomicroscopy on day 7 after implantation. To analyze whether the effect of cytokines on angiogenesis was direct or due to infiltration by inflammatory cells, histological studies were performed on sections after hematoxylin-eosin staining.

\section{Tissue preparation and histologic examination}

Immediately following the removal of human aortic segments (6 aortic dissections with atherosclerotic change and 6 aortic aneurysms with atherosclerotic change, median age; 54 years old) excluding dissection and aneurysmal segment, each segment was perfused with 
$10 \%$ formalin to maintain vascular integrity. The atherosclerotic change was confirmed microscopically. Two normal aortic segments obtained by surgical repair for traumatic aortic dissection were used as control (mean age: 28 years old).

Paraffin sections $(5 \mu \mathrm{m})$ from human aortas and rat cornea were made and transferred to slide glasses. Slides were deparaffinized and rehydrated. Endogenous peroxidase activity was blocked for $10 \mathrm{~min}$ at room temperature in $50 \%$ volume $\mathrm{H}_{2} \mathrm{O}_{2} / 50 \%$ volume methanol and rinsed in running tap water. Non-specific protein binding sites were blocked by applying $5 \%$ normal goat serum diluted in $\mathrm{PBS} / 0.05 \%$ Tween 20 (pH 7.2-7.4) to slides for $10 \mathrm{~min}$ at room temperature. The primary antibody (goat polyclonal antibodies for Ob-R; rabbit polyclonal antibodies for VEGF; goat polyclonal antibodies for MMP-2; goat polyclonal antibodies for TIMP-2; rabbit polyclonal antibodies for flk-1, Santa Cruz Biotechnology, Inc., Santa Cruz, CA, USA) was diluted in $1 \%$ normal goat serum in PBS $/ 0.05 \%$ Tween 20 , applied and incubated overnight at $4^{\circ} \mathrm{C}$ in a humidity chamber. Antibodies were directed against the carboxyl (C-20) or the amino terminus ( $\mathrm{N}-20)$ of $\mathrm{Ob}-\mathrm{R}$ recognized a $170 \mathrm{kD}$ protein, the size of which is consistent with the human leptin receptor. On day 2, the primary antibody was rinsed off in tap water, blotted and the biotinylated secondary antisera cocktail including goat anti-mouse $\operatorname{lgG}$ and goat anti-rabbit diluted 1/400 was incubated on the slides for $30 \mathrm{~min}$ at room temperature. Slides were rinsed in running tap water, blotted and streptavidinhorseradish peroxidase diluted $1 / 500$ in PBS/0.05\% Tween 20 containing $1 \%$ normal goat serum was applied and incubated for $30 \mathrm{~min}$ at room temperature. The slides were rinsed in tap water and color developed in 3-amino-9-ethylcarbazole substrate solution for 15 min at room temperature, then counterstained in methylene blue for $30 \mathrm{~s}$ and covered with coverslip.

\section{Statistical tests}

Significance of values were determined with the 2-tailed student's $t$ test. All results are expressed as the mean \pm SEM.

\section{Results}

\section{Cell proliferation study}

The effect of leptin on HUVEC and HCASMC proliferation was shown in Figure 1. Treatment with leptin (10 to $40 \mathrm{ng} / \mathrm{ml}$ ) stimulated HUVECs proliferation, but had no significant effect on HCASMCs proliferation. These differential effects of leptin on HUVECs and HCASMCs are similar to the effect of VEGF.

\section{In vitro angiogenesis assay}

As shown in Figure 2, HUVECs cultured on Matrigel containing leptin $(100 \mathrm{ng} / \mathrm{ml})$ were stretched and elongated forming a capillary-like structure within $4 \mathrm{~h}$, but HUVECs on untreated gel failed to form the capillary structure.

\section{Effect of leptin on VEGF and bFGF expression}

The concentrations of VEGF and bFGF after stimulation with leptin (10 to $40 \mathrm{ng} / \mathrm{ml}$ ) for $48 \mathrm{~h}$ were measured to determine whether the angiogenic effect of leptin is dependent on the other growth factors. As shown in Figure 3, leptin enhanced the VEGF expression in a dosedependent manner from HUVECs, but not HCASMCs. There was no significant change of bFGF expression in both HUVEC and HCASMC (data not shown).

\section{Effect of leptin on MMPs protein expression}

MMP-2 and MMP-9 productions by HUVEC and HCASMC were analyzed using zymography. The activities of MMP-
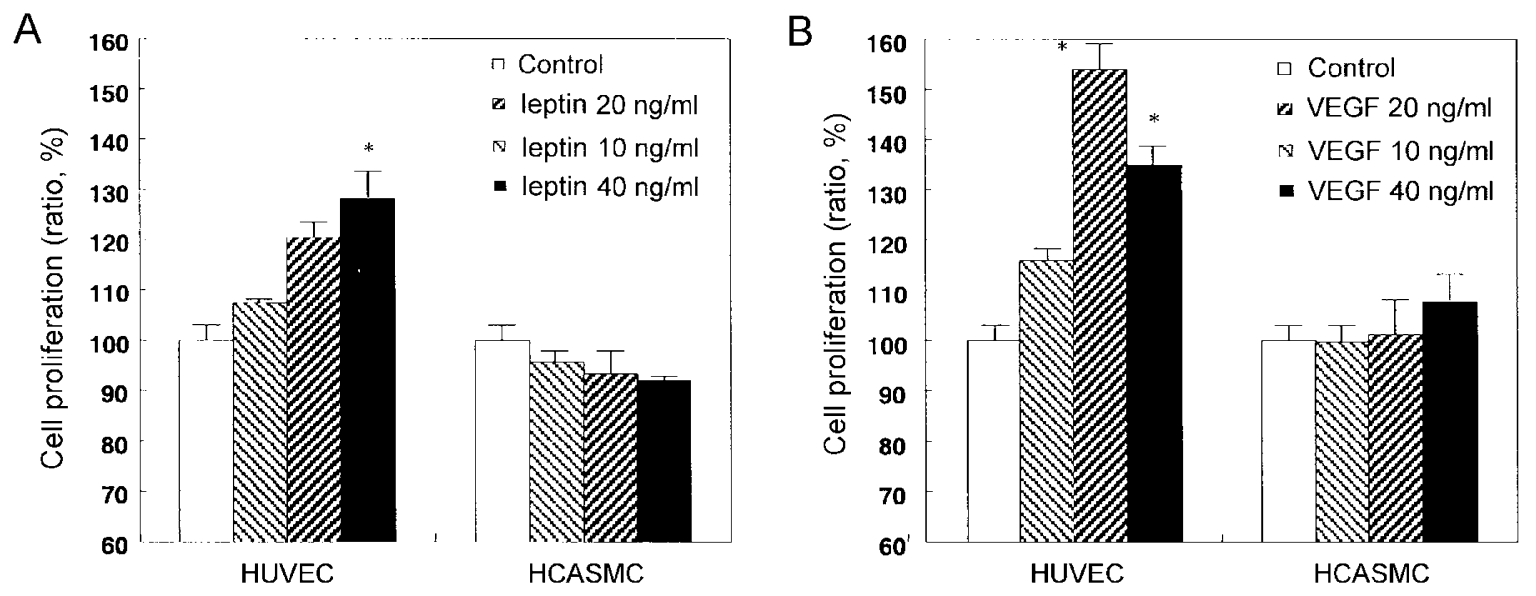

Figure 1. Effect of leptin on HUVECs and HCASMCs proliferation. HUVECs and HCASMCs were cultured in the presence of increasing concentration of human recombinant leptin $(\mathrm{A})$ or $\mathrm{VEGF}_{165}(\mathrm{~B})$ for $72 \mathrm{~h}$ in serum-deprived medium. Data are presented as mean $\pm \mathrm{SEM}$. Three independent experiments were performed. 
A

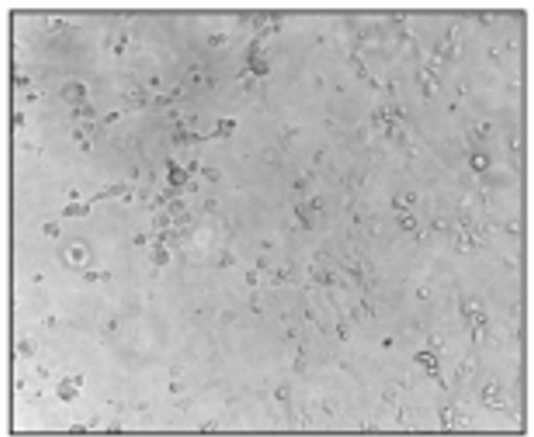

B

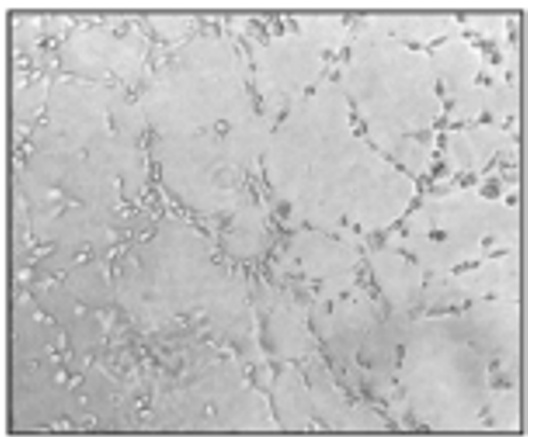

Figure 2. Effect of leptin on the formation of capillary-like structures in cultured HUVECs in growth factor-reduced Matrigel. HUVECs plated on Matrigel and cultured in serum free medium in the absence $(A)$ or presence of $100 \mathrm{ng} / \mathrm{ml}$ human recombinant leptin (B).

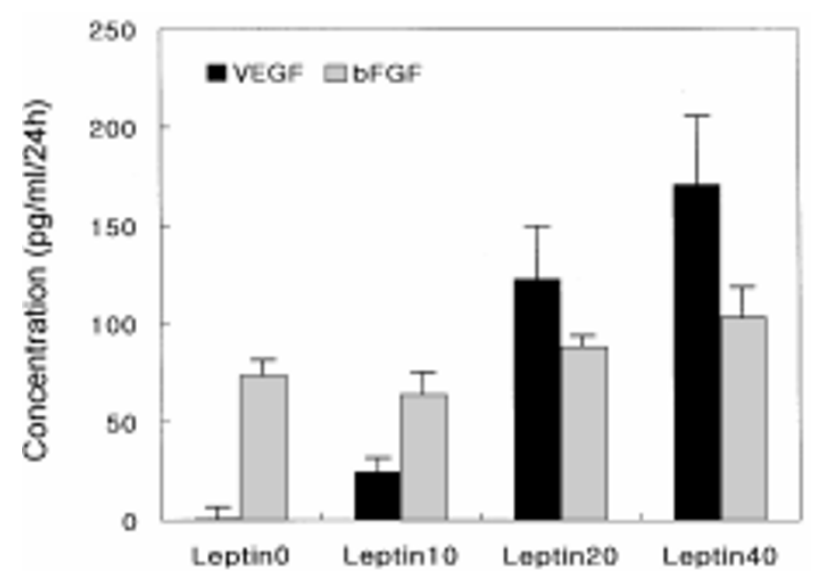

Figure 3. Effects of leptin on VEGF and bFGF secretion. Leptin upregulates the secretion of VEGF, but not bFGF by HUVECs.

2 and MMP-9 increased after treatment with leptin. The activities of pro- and active MMP-2 were increased 1.5fold at the leptin concentration of $10 \mathrm{ng} / \mathrm{ml}$, and 4-fold at a higher concentration (20 or $40 \mathrm{ng} / \mathrm{ml}$ ) in HUVECs (Figure 4A). Total amount of MMP-9 secretion was much lower than MMP-2 secretion and the effect of leptin on MMP-9 secretion was less prominent. The change of MMP-2 in HCASMCs was not as prominent as in HUVECs. However, at a high concentration of leptin $(40 \mathrm{ng} / \mathrm{ml})$, the activity of pro MMP-2 was increased as shown in Figure 4B.

\section{Effect of leptin on HCASMCs MMPs and TIMPs mRNA expression}

The mRNA levels of MMP-2, MMP-9, and TIMP-1 by HCASMCs were upregulated after leptin treatment (Figure $5 A$ ). The TIMP-2 protein expression by Western blot analysis was also increased in the HCASMCs treated with leptin (Figure 5B).

\section{Expression of Ob-R, VEGFR-1, and MMPs/TIMPs in neovascularized atheromatous plaque}

Control aortic tissues revealed little Ob-R (N-20, C-20) immunoreactivity in intimal SMCs and infiltrated inflammatory cells. The VEGFR-1 immunoreactivity was also weakly distributed in the endothelial lining with little nonendothelial immunoreactivity. Compared to the control aorta, the atherosclerotic aorta showed the increased cellularity accompanied by the infiltration of inflamma-
A

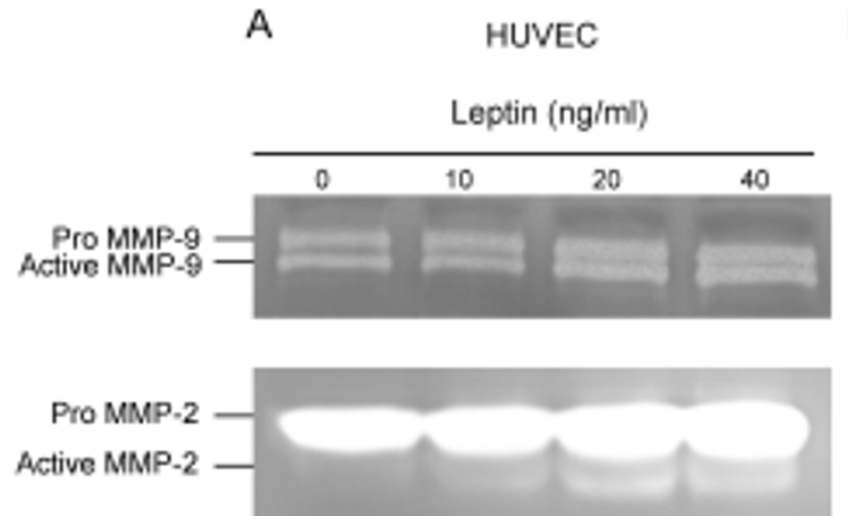

B

HCASMC

\begin{tabular}{cccc}
\multicolumn{4}{c}{ Leptin $(\mathrm{ng} / \mathrm{ml})$} \\
\hline 0 & 10 & 20 & 40 \\
\hline
\end{tabular}

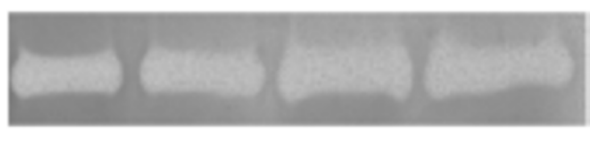

Figure 4. Effects of leptin on human MMP-2 and MMP-9 secretion. Leptin enhances MMP-2 secretion from both HUVECs and HCASMCs. Enzymatic activity of human MMP-2 and MMP-9 were analyzed using SDS-PAGE gelatin zymography. 
A

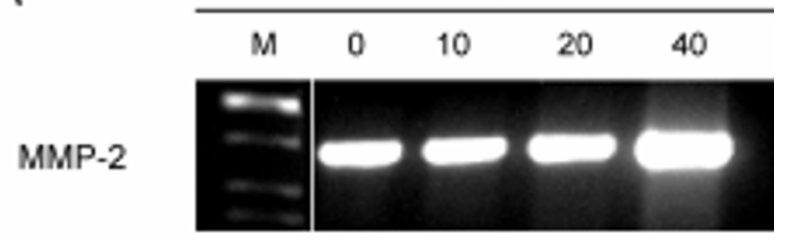

MMP-9

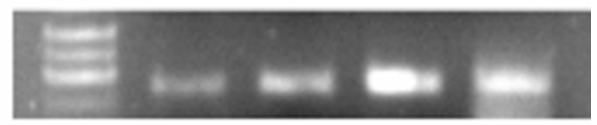

TIMP-1

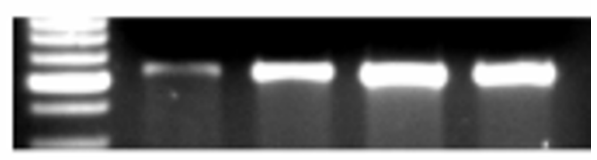

$\beta$-actin

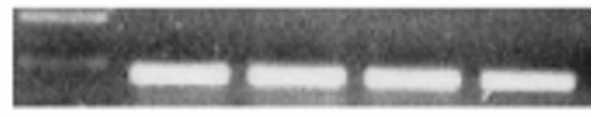

B

\begin{tabular}{llll}
\multicolumn{3}{l}{ Leptin $(\mathrm{ng} / \mathrm{ml})$} \\
\hline 0 & 10 & 20 & 40
\end{tabular}

TIMP-2

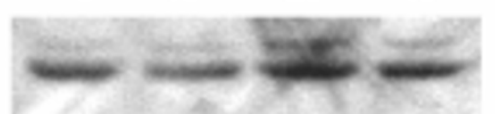

Figure 5. Effects of leptin on MMP-2, MMP-9, TIMP-1, and TIMP-2. The PCR products from CDNA derived by RT-PCR of HCASMCs with primers specific for MMP-2, MMP-9, and TIMP-1 were shown (A). Equal loading of RNA was conformed by the PCR products of $\beta$-actin. The expression of TIMP-2 was analyzed by the Western blot analysis (B).

tory cells, such as foam cells and neovascularization at plaque base (Figure 6A). Atherosclerotic aortic plaques demonstrated marked increase in Ob-R (C-20, N-20) immunoreactivity, predominantly in foam cells, VSMCs and the vascular endothelial lining of intimal neovessels (Figure 6B, C). Tissue expression of Ob-R (N-20, C-20) and VEGFR-1 were co-localized in atherosclerotic plaques especially in non-endothelial cells such as foam cells and vascular smooth muscle cells (VSMCs) (Figure 6D). Furthermore, immunoreactivity of Ob-R and VEGFR-1 were co-expressed within neovascular endothelial cells. The immunoreactivities for MMP-9 and TIMP-1 were evident in the neovascular endothelial cells suggesting that the expression of MMP has a role in the neovascularization in atheromatous plaque (Figure 6E, F).

\section{In vivo angiogenesis assays and immunohisto- chemistry}

The former results suggest that leptin induces angiogenesis through the endothelial proliferation and expression of MMPs. Thus, we used a rat corneal model to evaluate the angiogenic property of leptin in vivo and to investigate whether the expression of MMP is related with angiogenic activity of leptin. Leptin at a dose of 125 ng/pellet promoted a new vessel growth, progressing over half way to the lens by day 7 (Figure 7). Histologic studies stained with hematoxylin \& eosin demonstrated that rat cornea treated with Hydron pellet containing leptin revealed blood filled neovessels compared with rat corneae treated with PBS pellets. Comparing pellet with vehicle alone (Figure $8 \mathrm{~A}$ ), immunoreactivity of MMP-2, MMP-9, TIMP-2 and VEGFR-1 were markedly increased around the leptin-treated pellets (Figure 8B).

\section{Discussion}

In the present study, we have demonstrated the angiogenic activity of leptin and its possible mechanisms including indirect effect through the VEGF and effect on the MMP. Leptin, the product of the ob gene secreted by adipocytes, has been known to play a key role in the regulation of body weight, reproduction, hematopoiesis, and proinflammatory response (Bennett et al., 1996; Gainsford et al., 1996; Conway and Jacobs, 1997; Loffreda et al., 1998; Santos-Alvarez et al., 1999). Leptin acting as a functional link between adipocytes and the vasculature, might also play an important extrahypothalamic role in the modulation of adipose tissue mass. Recently, Sierra-Honigmann et al. (1998) demonstrated that treatment of HUVECs with leptin enhanced formation of capillary-like tubes in the in vitro angiogenesis assay and neovascularization in the in vivo model of angiogenesis. We hypothesized that the overexpression of leptin has a role in the growth of atheromatous plaques through its effect on neovascularization.

In atherosclerotic plaques, the immunoreactivity of $\mathrm{Ob}$ $R$ increased especially in foam cells and VSMCs. Interestingly, the immunoreactivity of VEGFR-1 was colocalized in atheromatous plaques and observed in foam cell-rich regions adjacent to the lipid core or the basal regions of plaque consisting predominantly of VSMCs. Ananyeva et al. (1997) recently demonstrated that ox$\mathrm{LDL}$ induced the release of aFGF from aFGF-transfected VSMCs. VEGF receptor (flt-1) expression in monocyte and VSMCs has recently been confirmed; this receptor mediates chemotaxis and tissue factor production associated with up-regulation of MMPs to contribute to the lateral expansion of new blood vessels. However, the immunoreactivities of Ob-R, MMPs and their inhibitors (TIMPs) increased in atherosclerotic plaques simultaneously with VEGFR-1, suggesting that the expression of VEGF is related with the increased activity of leptin. The experimental study with rat corneas also showed a prominent co-localization of $\mathrm{Ob}-\mathrm{R}$ and VEGFR-1 suggesting that the coupling of these factors 

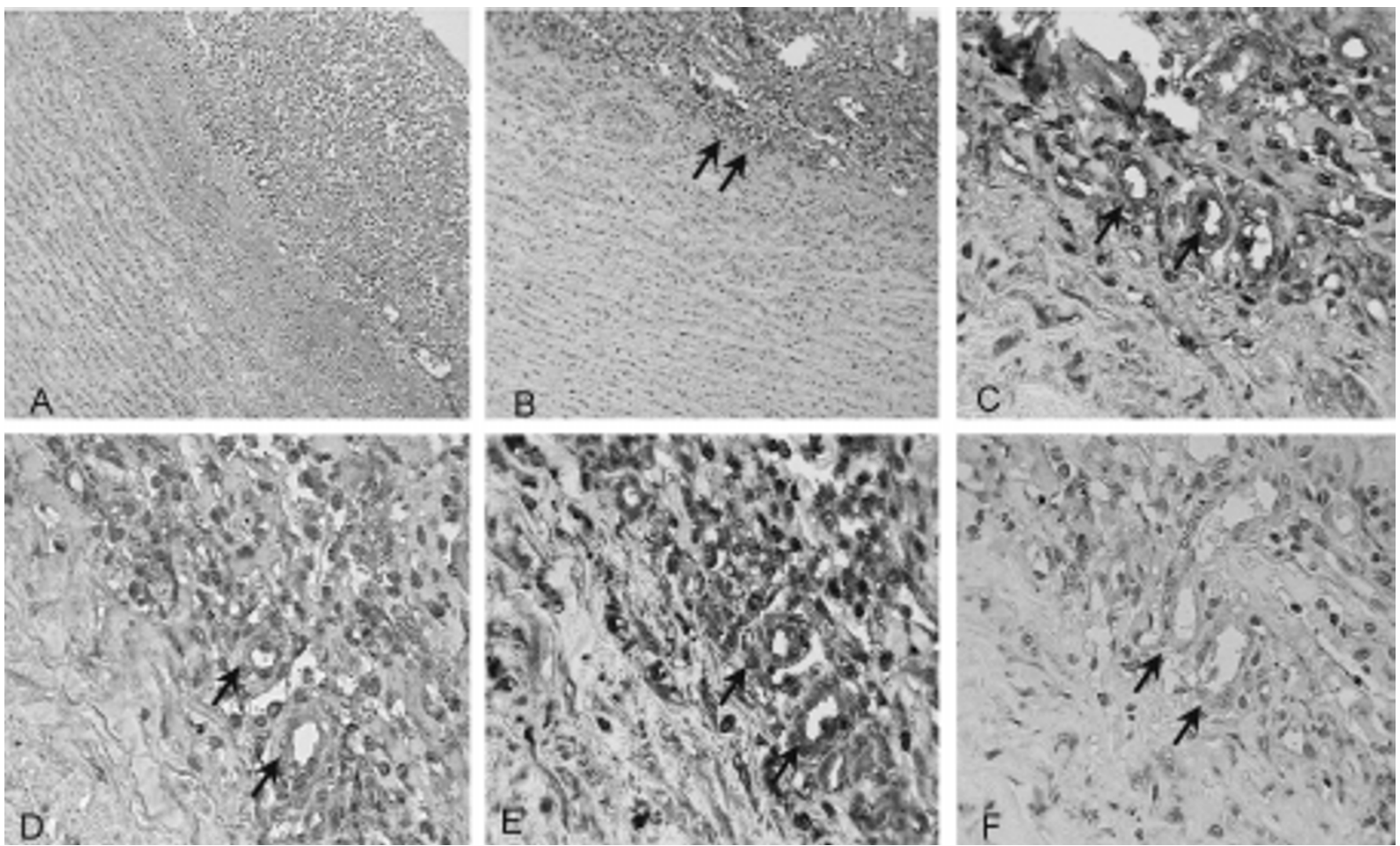

Figure 6. Hematoxylin \& eosin stain of atherosclerotic lesion (panel A; final magnification, $100 \mathrm{x}$ ). Expression of the leptin receptor using a goat polyclonal antibody directed against the amino (N-20)(panel B; $100 \mathrm{x}$ ), carboxyl (C-20)(panel C; $400 \mathrm{x}$ ) terminus of human Ob-R, immunoreactivity for VEGFR-1 (panel D; $400 \mathrm{x}$ ), MMP-9 and TIMP-1 (panel E and F; $400 \mathrm{x}$ ) in atherosclerotic lesion. A strong immunostaining was seen in atherosclerotic plaque, mainly in foam cells, vascular smooth muscle cells and the vascular endothelial lining of intimal neovessels (black arrows).
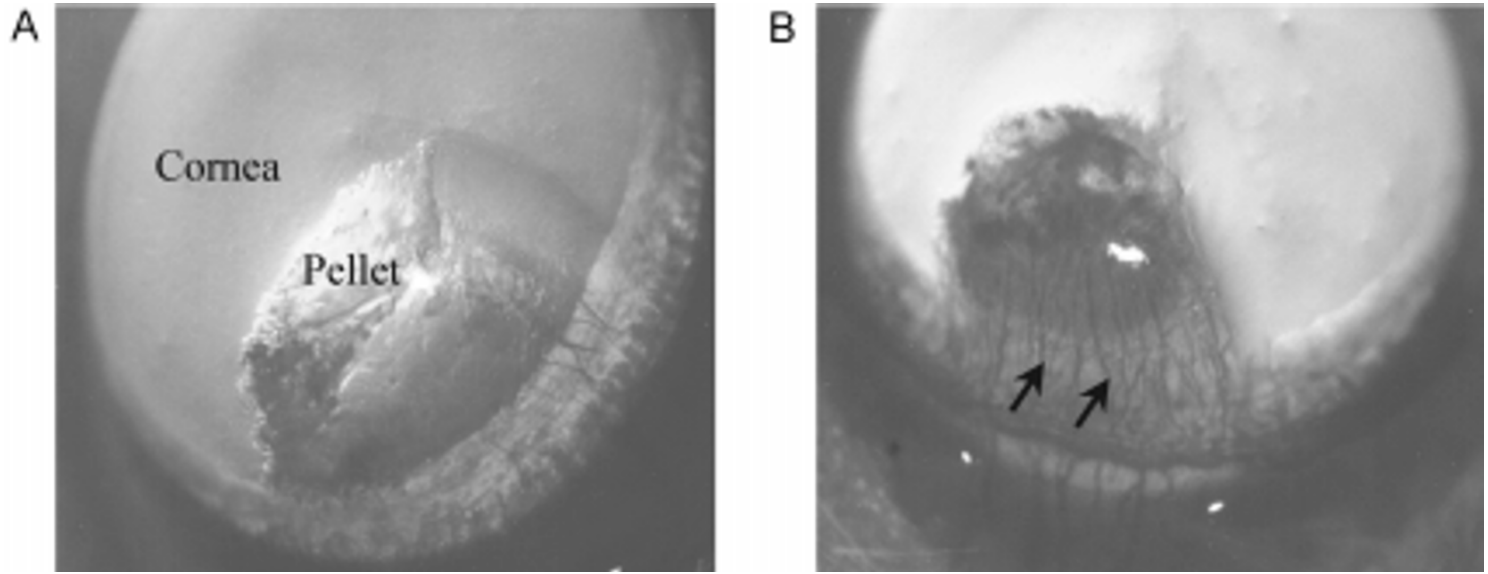

Figure 7. In vivo angiogenic activity of leptin. Corneal response 7 days after implantation of a Hydron pellet containing PBS (A) or $125 \mathrm{ng}$ of leptin (B). Arrow indicates neovessels progressing from the limbus to the pellet.

participates in neovascularization. The in vitro study used to elucidate the effect of leptin on VEGF and bFGF expression showed that leptin increased the secretion of VEGF in HUVECs in a dose-dependent manner, but it had little effect on the secretion of bFGF. However, leptin has little effect on the secretion of VEGF ${ }_{165}$ or bFGF in HCASMC. The differential effects on VEGF according to the cell type need a further study.
One of the initial steps in the angiogenesis process involves the degradation of the subendothelial basement membrane and surrounding extracellular matrix (ECM). Following matrix breakdown, endothelial cells migrate and proliferate to form new vessels, and proteolytic enzymes that degrade ECM mediates this process. The MMPs, a family of zinc-dependent proteinase, can digest various ECM components, the requirement for 
A
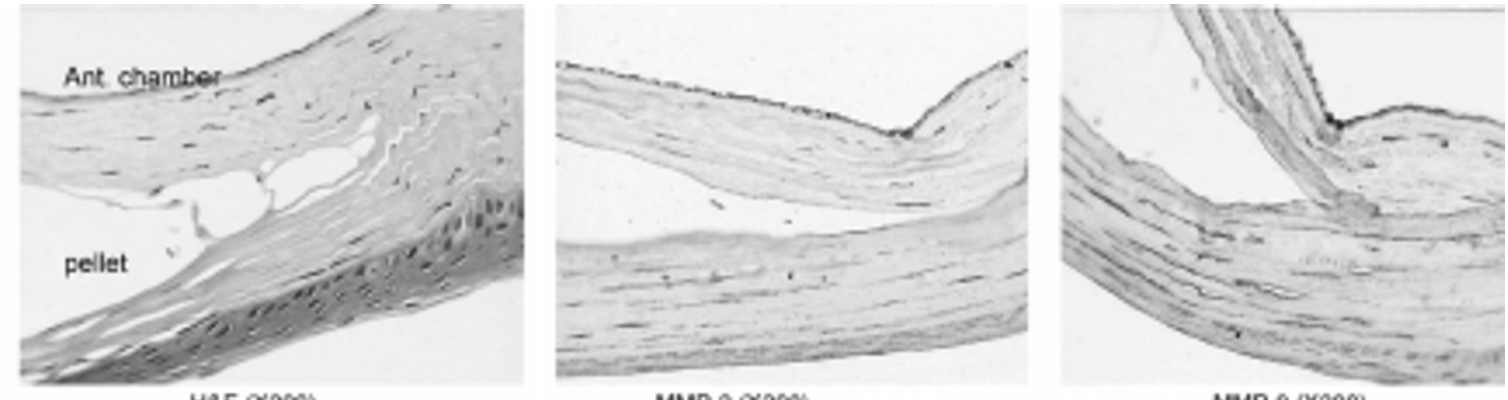

$M M P-2(2000)$

MUP-9 $(X 200)$

B
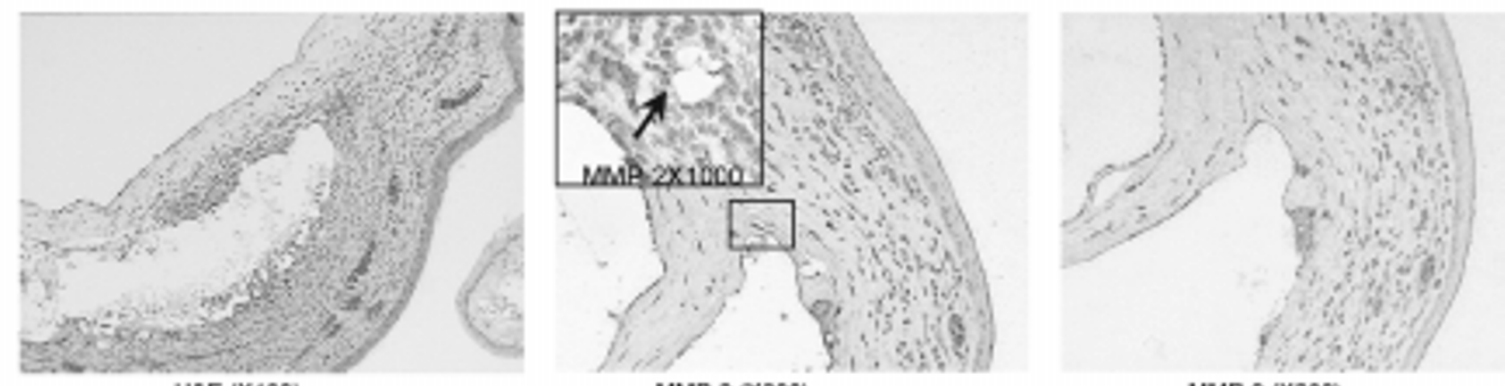

HSE $\langle\mathrm{X} 100\rangle$
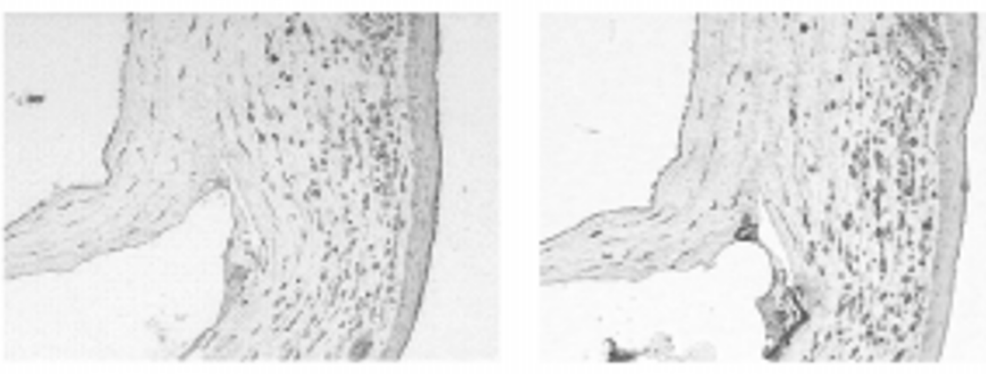

$\operatorname{TMP}-2\langle X 200\}$

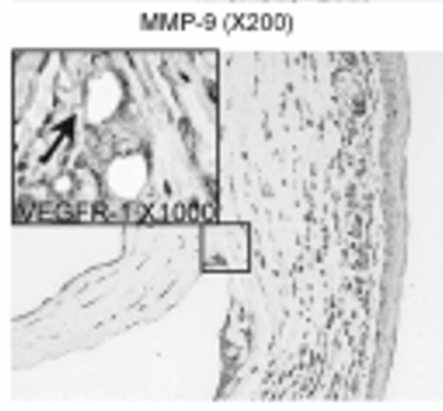

VEGFR-1 〈X200)

Figure 8. Immunohistochemical staining was performed with antibody for MMP-2, MMP-9, TIMP-1, TIMP-2, and VEGFR-1 (flt-1) in rat cornea on day 7 after implantation of a Hydron pellet containing PBS (A) or $125 \mathrm{ng}$ of leptin (B). Comparing with Hydron pellet containing PBS, rat cornea with leptin-treated pellet reveals blood filled neovessels. Immunoreactivity for MMP-2, MMP-9, TIMP-2 and VEGFR-1 are markedly increased around the pellets containing leptin. Arrows indicate neovessels. Lacunae in corneal tissue represent the sections of vessels. In controls, only a few vessels are noted, whereas in leptin-treated animals, marked angiogenesis is obvious.

cellular migration. Wang and Keiser (1998) demonstrated the enhancement of MMP expression in arterial SMCs after VEGF stimulation. The vascular localization of MMP may permit their spatial role in the degradation of subendothelial basement membrane, which is a crucial step in angiogenesis and atherosclerosis (StetlerStevenson, 1999). MMPs are involved in more than the breakdown of connective tissue barriers necessary for new vessel formation. They also function to promote angiogenesis by regulating endothelial cell attachment, proliferation and migration of endothelial cells, either directly or by release of growth factors sequestered in the ECM. Moreover, new evidence suggests that MMPs may also generate or release angiogenesis inhibitors such as angiostatin from the ECM (Cornelius et al., 1998). Among MMPs, MT-1-MMP and MMP-2 are active on the endothelial cell surface and function immediately in extracellular matrix turnover and regulation of cell-
ECM interactions. These changes in turnover of the ECM may result in phenotypic changes associated with the angiogenic response (Stetler-Stevenson, 1999). TIMP-2 binding to the active site of MT-1-MMP and the $\mathrm{COOH}$ terminal hemopexin-like domain of pro-MMP-2 results in the formation of a ternary complex. If this occurs in proximity to a second MT-1-MMP molecule, proteolytic modification of the pro-fragment of MMP-2 initiates an activation of this protease. The activated protease may then dissociate from the cell surface and contribute to degradation of the extracellular matrix prerequisite for endothelial sprout invasion. Although recent studies suggest the expression of leptin receptor in cell culture study and nonvascular tissue, there was no information about the interaction between leptin and MMPs/TIMPs (Bouloumié et al., 1998; Sierra-Honigmann et al., 1998).

In the present study, we demonstrated that the leptin receptor was expressed in the human atheromatous 
plaques with neovascularization using polyclonal antibodies to synthetic peptides based on the sequence of human leptin receptor and leptin induced the proliferation of endothelial cell and expression of MMPs/ TIMPs. In vitro study, the leptin increased the secretion of VEGF in HUVECs in a dose dependent manner and treatment with leptin on HUVECs and HCASMCs enhanced the expression of MMP-2, MMP-9, TIMP-1, and TIMP-2 by Western blotting and RT-PCR. These findings suggested that the leptin might be used as an angiogenic factor or mitogenic factor of vascular endothelial cells and play a role in matrix remodeling of angiogenic process and on neovascularization microenvironment. The present study might open a promising perspective in regard to the future investigations of leptin-MMPs dependent modulation of atherogenesis and vascular neovascularization under pathophysiological conditions.

\section{Acknowledgement}

This work is supported by the Yonsei University, College of Medical Research grants in 2000 (2000-6) and supported by BK21 Project for Medical Science, Yonsei University. We thank J.B. Lee for expert technical assistance in animal experiment. We are grateful to H.W. Yang for a critical reading of the manuscript.

\section{References}

Ananyeva NM, Tjurmin AV, Berliner JA, Chisolm GM, Liau G, Winkles JA, Haudenschild CC. Oxidized LDL mediates the release of fibroblast growth factor-1. Arterioscler Thromb Vasc Biol 1997; 17:445-53

Bennett B, Solar G, Yuan J, Mathias J, Thomas G, Matthews W. A role for leptin and its cognate receptor in hematopoiesis. Curr Biol 1996;6:1170-80

Bouloumié A, Drexler HCA, Lafontan M, Busse R. (1998) Leptin, the product of $\mathrm{Ob}$ gene, promotes angiogenesis. Circ Res 1998;83:1059-66

Campfield L, Smith F, Guisez Y, Devos R, Burn P. (1995) Recombinant mouse $\mathrm{OB}$ protein: evidence for a peripheral signal linking adiposity and central networks. Science 1995;
269:546-49

Conway G, Jacobs H. Leptin:a hormone of reproduction. Hum Reprod 1997;12:633-35

Cornelius LA, Nehring LC, Harding E, Bolanowski M, Welgus HG, Kobayashi DK, Pierce RA, Shapiro SD. Matrix metalloproteinases generate angiostatin: Effect on neovascularization. The Journal of Immunology 1998;161:6845-52

Gainsford T, Willson T, Metcalf D, Handman E, McFarlane C, $\mathrm{Ng} \mathrm{A}$, Nicola N, Alexander W, Hilton D. Leptin can induce proliferation, differentiation, and functional activation of hematopoietic cells. Proc Natl Acad Sci USA 1996;93:1456468

Halaas JL, Gajiwala KS, Maffei M, Cohen SL, Chait BT, Rabinowitz D, Lallone RL, Burley SK, Friedman JM. Weightreducing effects of the plasma protein encoded by the obese gene. Science 1995;269:543-46

Kahlon R, Shapero J., Gotlieb Al. Angiogenesis in atherosclerosis. Can J Cardiol 1992;8:60-64

Loffreda S, Yang SQ, Lin HZ, Karp CL, Brengman ML, Wang DJ, Klein AS, Bulkley GB, Bao C, Noble PW, Lane MD, Diehl AM. Leptin regulates proinflammatory response. FASEB J. 1998;12:57-65

O'Brien ER, Garvin MR, Dev R, Stewart DK, Hinohara T, Simpson JB, Schwartz SM. Angiogenesis in human coronary atherosclerotic plaques. Am J Pathol 1994;145:883-94

Pelleymounter MA, Cullen MJ, Baker MB, Hecht R, Winters D, Boone T, Collins F. Effects of the obese gene product on body weight regulation in ob/ob mice. Science 1995;269:540-43

Santos-Alvarez J, Goberna R, Sanchez-Margalet V. Human leptin stimulates proliferation and activation of human circulating monocytes. Cell Immunol 1999;194:6-11

Sierra-Honigmann MR, Nath AK, Murakami C, GarciaCardena G, Papapetropoulos A, Sessa WC, Madge LA, Schechner JS, Schwabb MB, Polverini PJ, Flores-Riveros JR. Biological action of leptin as an angiogenic factor. Science 1998;281:1683-86

Stetler-Stevenson WG. Matrix metalloproteinase in angiogenesis: a moving target for therapeutic intervention. J Clin Invest 1999;103:1237-41

Wang $\mathrm{H}$, Keiser JA. Vascular endothelial growth factor upregulates the expression of matrix metalloproteinases in vascular smooth muscle cells: role of flt-1. Circ Res 1998;83: $832-40$ 